\title{
A New Slip Stacking RF System for a Twofold Power Upgrade of Fermilab's Accelerator Complex
}

\author{
R. Madrak \\ Fermi National Accelerator Laboratory, P.O. Box 500, Batavia, IL 60510-5011, USA.
}

\begin{abstract}
Fermilab's accelerator complex has been recently upgraded, in order to increase the $120 \mathrm{GeV}$ proton beam power on target from about $400 \mathrm{~kW}$ to over $700 \mathrm{~kW}$ for $\mathrm{NO} v \mathrm{~A}$ and other future intensity frontier experiments. One of the key ingredients of the upgrade is the offloading of some Main Injector synchrotron operations - beam injection and RF manipulation called "slip stacking" - to the $8 \mathrm{GeV}$ Recycler Ring, which had until recently been used only for low-intensity antiproton storage and cooling. This required construction of two new $53 \mathrm{MHz}$ RF systems for the slip-stacking manipulations. The cavities operate simultaneously at $V_{\text {peak }} \lesssim 150 \mathrm{kV}$, but at slightly different frequencies $(\Delta f=1260 \mathrm{~Hz})$. Their installation was completed in September 2013. This paper describes the the novel solutions used in the design of the new cavities, their tuning system, and the associated high power RF system. First results showing effective operation of the RF system, beam capture and successful slip-stacking in the Recycler Ring are presented.
\end{abstract}

Keywords: RF cavities, slip stacking, Recycler Ring, $53 \mathrm{MHz}$

\section{Introduction}

In recent years, with the retirement of the Tevatron proton-antiproton collider [1] [2] and the conclusion of the collider physics program, the main focus at Fermi National Accelerator Laboratory (FNAL, Fermilab) has shifted from energy frontier research to intensity frontier research. The goal is still to uncover new physics, but the method

Email address: madrak@fnal.gov (R. Madrak) 
differs. As an alternative to searching for new particles at increasingly high energies, the approach is through precise measurements of certain particle interactions; this requires large data samples. Many such examples are seen in neutrino physics, as well as in other areas of study such as rare decays and rare processes in muons, but all require more protons on target. Thus, the goal is and will continue to be increasing proton beam intensity.

The FNAL Accelerator Complex, with its multitude of experiments, is shown in Fig. 1. Several plans are in progress for increasing proton power by upgrades to the Booster [3] [4] and Linac [5]. Here, we concern ourselves with an upgrade and repurposing of the existing Recycler Ring (RR), which was until recently used for antiproton storage for the collider program. This will ultimately increase proton output at $120 \mathrm{GeV}$ from the Main Injector (MI) and protons on target for the newly constructed neutrino experiment $\mathrm{NO} v \mathrm{~A}[6]$. $\mathrm{NO} v \mathrm{~A}$ will study the neutrino oscillation $v_{\mu} \rightarrow v_{e}$ with near and far detectors off-axis from the existing MINOS [7] beamline.

Until May 2012, the process for $120 \mathrm{GeV}$ proton production included injection and capture of Booster batches, slip stacking, and acceleration - all performed in the MI. (The Booster has harmonic number $h=84$. A batch consists of 81 bunches which have been accelerated to $8 \mathrm{GeV}$ in the Booster.) Now, injection/slip stacking will be performed in the RR and acceleration in the MI so that the two processes can proceed in parallel. In this case, the $120 \mathrm{GeV}$ proton production rate is limited by only the MI ramp time (since the ramp time is longer than the time to capture and slip stack). Also, the MI ramp rate will be increased by $15 \%$. This results in an average MI beam power increase from $\approx 400 \mathrm{~kW}$ to $\approx 700 \mathrm{~kW}[8]$.

To perform slip stacking in the RR, two new $53 \mathrm{MHz}$ RF cavities and associated RF systems were designed and constructed (a third, spare system is also nearing completion). Since the cavities need not both capture beam and accelerate, they were designed expressly for the former purpose. This allowed for the minimization of $R / Q$ and thus transient beam loading effects [9], while taking into account practical constraints, such as being able to fit in the tunnel given the RR beampipe position with respect to the tunnel ceiling.

In addition to repurposing the RR itself, many components from the Tevatron RF 


\section{Fermilab Accelerator Complex}

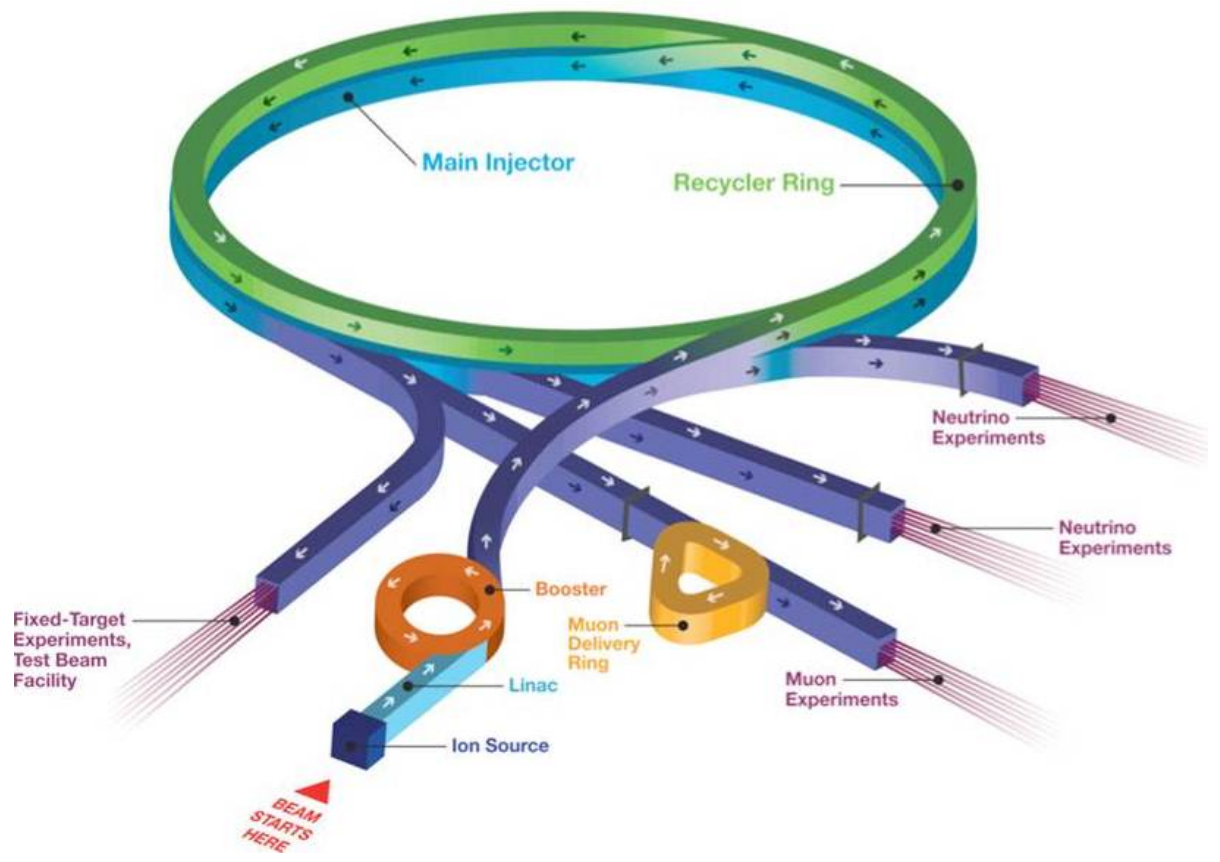

Figure 1: The FNAL Accelerator complex. In this paper, we focus on the Recycler Ring, which captures and slip stacks $8 \mathrm{GeV}$ Booster beam. After the manipulations in the Recycler, beam is sent to the Main Injector for acceleration from 8 to $120 \mathrm{GeV}$.

systems were re-used for the new RF systems. This was possible since the Tevatron RF system operated at a similar frequency and at similar power levels. Reclaimed apparatuses include modulators, power amplifiers (with tetrodes), solid state amplifiers for driving the power amplifiers, coaxial waveguide and associated components, and various electronic control modules. The obvious advantage is savings in cost, labor and waste.

As is the case in most RF systems, one of the more challenging aspects of the system design is in the cavity tuner. A large fraction of conventional (non superconducting) RF cavities in operation require a large tuning range and make use of parallelbiased ferrite. In the case of the cavities discussed here, the required tuning range is just $10 \mathrm{kHz}$, which allows use of the less lossy perpendicularly biased garnet. 


\section{Slip Stacking}

The Booster magnets cycle at a rate of $15 \mathrm{~Hz}$. The magnet system is resonant so the ramp is sinusoidal and the ramp time is not adjustable. Due to limitations imposed by the current system for RF cavity cooling, not all cycles accelerate beam and the resulting average rate with beam is $7.5 \mathrm{~Hz}$.

In the simplest of schemes, $120 \mathrm{GeV}$ proton production could proceed by simply injecting six batches from the Booster into the MI and then ramping the MI (i.e. accelerating from 8 to $120 \mathrm{GeV}$ ). The nominal MI RF system injection frequency is $52.809 \mathrm{MHz}$ with $588 \mathrm{RF}$ buckets (harmonic number $h=588$ ), so its circumference can technically accommodate seven Booster $(h=84)$ batches, but an abort gap must exist. Assuming that the first of the six Booster batches is ready on demand, injection takes 5 batches $\times(1 / 15 \mathrm{~Hz})=1 / 3 \mathrm{sec}$. After the injection, the beam is accelerated. The MI cycle time excluding injection is $1.533 \mathrm{sec}$. Adding the additional $1 / 3 \mathrm{sec}$ for injection, six Booster batches are accelerated to $120 \mathrm{GeV}$ in $1.87 \mathrm{sec}$.

A more efficient scheme, which has been the norm for operations since 2008, makes use of a process called slip stacking [10], which was initially demonstrated at CERN[11]. For acceleration, the MI normally uses 18 cavities operating at $V_{\text {peak }} \approx 230$ $\mathrm{kV}$. For slip stacking, the cavities are run at a lower voltage, and are divided into RF Systems A and B, each of which comprises nine cavities. Five Booster batches are injected into the MI at its nominal frequency $f_{0}$, captured by RF System A operating at $f_{0}$, and then decelerated slightly by changing the frequency of RF System A by $1260 \mathrm{~Hz}$. While the first five batches that have been captured continue to circulate, five more batches are injected and captured by RF system B, operating at $f_{0}$. Since the initial five batches have been captured by an RF system which is now operating at a lower frequency, they slip in phase with respect to the second five batches. When the first and second set of batches have slipped with respect to one another so that they overlap in their azimuthal positions, both RF systems revert to operation at $f_{0}$. An 11th batch is injected, voltage is increased to $1 \mathrm{MV}$ for capture, and the beam is finally accelerated. The accelerated beam consists of five batches having been stacked on top of five batches (plus one extra), thus the term slip stacking. In this case, 11 batches of beam 
are accelerated to $120 \mathrm{GeV}$ in $2.2 \mathrm{sec}(0.667 \mathrm{sec}$ for injection/stacking and $1.533 \mathrm{sec}$ for the actual dipole magnet ramp), so the beam power is $\approx 50 \%$ higher than in the case described previously. Note that the initial frequency difference of $1260 \mathrm{~Hz}$ is set by the fact that that one batch ( 84 proton bunches) must slip by 84 RF buckets during one booster cycle of $66.7 \mathrm{~ms}(1 / 15 \mathrm{~Hz})$, so 84 cycles $/ 66.7 \mathrm{~ms}=1260 \mathrm{~Hz}$.

To achieve even higher beam powers, this concept was extended further by repurposing the existing RR, which, until the conclusion of the collider program, had been used for antiproton storage. By using the RR as a preinjector and performing slip stacking there while using the MI for acceleration only, the two processes can proceed in parallel and the rate is only limited by the MI cycle time. As mentioned earlier, the MI will also be upgraded to decrease its cycle time from 1.533 to $1.33 \mathrm{sec}$. In addition, 12 instead of 11 batches will be slip stacked in the RR; this is allowed for by the installation of a fast injection kicker. The result is 12 Booster batches of $120 \mathrm{GeV}$ protons produced in $1.33 \mathrm{sec}$, compared to 11 batches in $2.2 \mathrm{sec}$ with slip stacking and acceleration in the MI.

\section{Cavity Design}

Slip stacking in the RR uses two cavities operating at the MI injection frequency of $52.809 \mathrm{MHz}$ and $V_{\text {peak }} \approx 100 \mathrm{kV}$. Including a safety factor, the cavities were designed for $V_{\text {peak }}=150 \mathrm{kV}$. The cavities must be tunable by $1260 \mathrm{~Hz}$ for slip stacking manipulations. Given other design parameters which are discussed next, the actual required tuning range is $10 \mathrm{kHz}$, to compensate for frequency drift due to heating and the variation of cooling water temperature. (The cavity detuning was measured to be $\approx$ $0.5 \mathrm{kHz} /{ }^{\circ} \mathrm{F}$.) The maximum pulse length is $0.8 \mathrm{sec}$, given by the time required to stack 12 Booster batches. The maximum duty factor is 0.6 , assuming that the cavities are on for a total of $0.8 \mathrm{sec}$ for each MI ramp of $1.33 \mathrm{sec}$ and that the MI ramps continuously.

The cavities are quarter wave resonators with coaxial center and outer conductors of fixed diameter. The design is shown in Fig. 2.

Cavity parameters are summarized in Table 1 . In the interest of minimizing transient beam loading, the cavity geometry was chosen to minimize $R / Q$ within the con- 


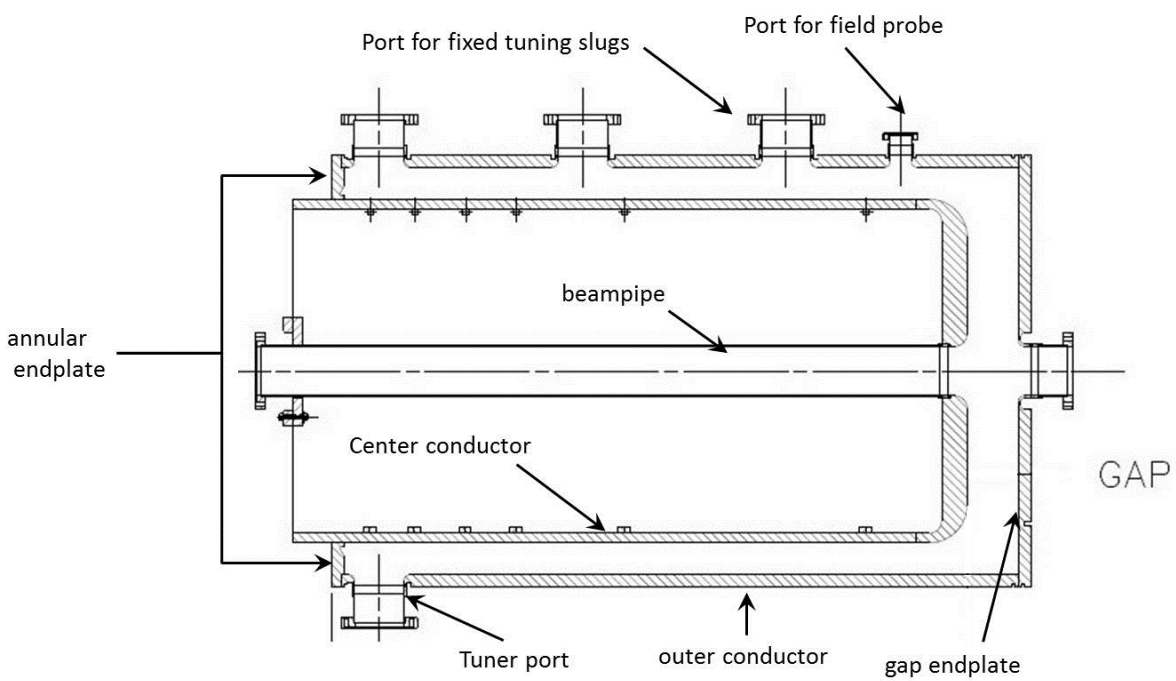

Figure 2: Side view of the quarter wave cavity design. The center conductor is shorted to the outer conductor by an annular endplate. A peak voltage of $150 \mathrm{kV}$ is generated across the gap.

straints of tunnel size and shunt impedance $\left(R_{s h}\right)$. With this geometry [12]

$$
Z_{0}=60 \ln \left(r_{\text {outer }} / r_{\text {inner }}\right) \text { and } R / Q \approx(4 / \pi) Z_{0}
$$

where $Z_{0}$ is the characteristic impedance of the coaxial transmission line formed by the cavity inner and outer conductors. The tunnel space restricted the inner diameter (ID) of the cavity outer conductor to 32 inches, taking into account available space and allowances for ports and connections. For the required $V_{\text {peak }}$ of $150 \mathrm{kV}$ with $150 \mathrm{~kW}$ of power available from the repurposed Tevatron RF systems, $R_{s h}$ must be at least $75 \mathrm{k} \Omega$. The $R / Q$ of $13 \Omega$ is substantially smaller than that of the MI cavities (103 $\Omega$ ) due mainly to the small ratio of diameters of the outer and center conductors.

For calculations, the cavity can be modeled as a shorted transmission line whose inductance and gap capacitance form a resonant circuit, as discussed in [12]. The initial design and calculations were checked by constructing a model of the cavity from copper sheet metal [13]. Though such a cavity is not useful for any high power tests, it established a lower limit on the cavity $Q$ and was useful for the study of higher order modes and development of the cavity tuner. 


\begin{tabular}{l|r}
\hline \hline$f_{0}$ & $52.809 \mathrm{MHz}$ \\
\hline$V_{\text {peak }}(\mathrm{max})$ & $150 \mathrm{kV}$ \\
\hline Maximum power & $150 \mathrm{~kW}$ \\
\hline$R_{s h}$ & $75 \mathrm{k} \Omega$ \\
\hline$R / Q$ & $13 \Omega$ \\
\hline$Z_{0}$ & $10.2 \Omega$ \\
\hline$Q$ (measured) & 5800 \\
\hline Outer conductor ID & 32 in \\
\hline Inner conductor OD & $27 \mathrm{in}$ \\
\hline nominal gap width & $2.9 \mathrm{in}$ \\
\hline Inner conductor length & $49.75 \mathrm{in}$ \\
\hline Wall thickness & 1 in \\
\hline
\end{tabular}

Table 1: Cavity Design Parameters

\subsection{Cavity Accessories}

The cavity design includes ports of various sizes for the input coupler, field probes, fixed tuning slugs, and a cavity shorting system. The coupler is a loop type with a voltage step-up ratio of six. Field probes are disk-shaped and capacitively coupled, with a nominal voltage ratio of $10^{5}: 1$. Fixed copper tuning slugs are inserted into the cavity ports for the final adjustment of the cavity nominal frequency. To render a cavity inactive (for instance, in the case where the spare is being used), each cavity is equipped with a copper shorting slug remotely controlled by a stepper motor.

For the frequency adjustment needed for slip stacking, and to account for detuning due to temperature change, the cavity is equipped with a tuner. This is a halfwavelength $50 \Omega$ transmission line, which is partially loaded with a $5^{\prime \prime}$ long cylinder of Al doped Yttrium Iron Garnet (YIG) - TCI Ceramics Type AL-400 and shorted at one end. The garnet section is immersed in a variable solenoidal magnetic field. The tuner is loop coupled to the cavity and the loop area is adjusted to give a nominal coupling impedance of $50 \Omega$. It is discussed in detail in Section 6 . 


\subsection{RF Power}

The Fermilab Tevatron has been decommissioned and thus many of the components of the Tevatron RF Stations could be reused; this includes the power amplifiers, anode modulators, solid state tetrode drivers, $9 \frac{3}{16}$ " coaxial transmission line, and impedance matching anode resonators. The Tevatron RF systems used essentially the same power amplifiers as does the MI, and these are described in [14]. Power is provided by a grounded grid, cathode driven Eimac Y567B (4CW150000E) 150kW power tetrode. The cathode is driven by solid state $5 \mathrm{~kW}$ amplifiers. The tetrode is coupled with a monolithic ceramic blocking capacitor to a cylindrical aluminum quarter wave resonator (the anode resonator) which is used to match the power amplifier output impedance into a $9 \frac{3}{16}{ }^{\prime \prime}, 50 \Omega$ transmission line. The tap point is at one half the peak voltage. The transmission line includes a variable length phase shifter and a 3:1 coaxial transformer with $1 \frac{1}{2}$ wavelengths between the transformer voltage maximum and the cavity input. Taking into account the cavity input coupler step-up ratio of 6 , the 3:1 transformer, and the half voltage tap point on the anode resonator, the total step up ratio from the anode to the cavity gap is nine.

\section{Cavity Construction}

The main body of the cavity consists of four pieces: the center conductor, the outer conductor, the annular region connecting the center to the outer, and the gap endplate (refer to Fig.2).

The cavity center and outer conductors were forged at Scot Forge, Spring Grove, IL from oxygen free high conductivity (OFHC) copper ingots. The outer conductor was forged into a hollow cylinder with $>1$ inch thick walls. The inner conductor was forged into a cup shape. Following the forging they were machined to final dimensions and ports were added. The circular and annular regions which form the ends of the cavity were also machined from solid blocks of oxygen OFHC copper. The OFHC copper was chosen because of concerns about welding (porosity and brittleness), not because it has slightly higher conductivity than standard copper. 


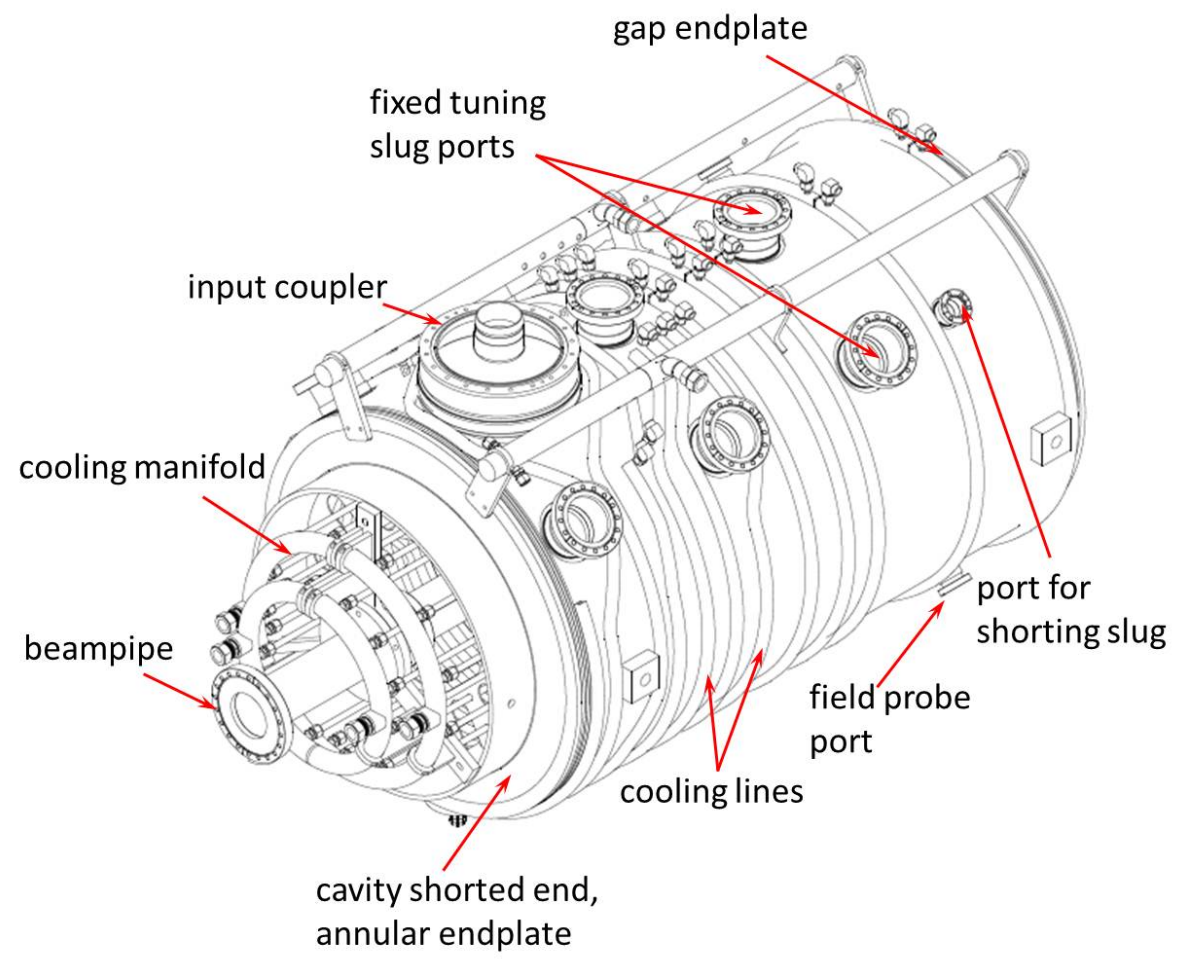

Figure 3: Three dimensional view of the cavity from the shorted end.

The main cavity components were electron beam welded at Sciaky Inc., Chicago, IL. The electron beam welds are between the inner conductor to the annular region, between the outer conductor to the annular region, and between the gap endplate to the outer conductor. The cavity frequency was tuned after welding the center conductor to the annular ring by adjusting (by machining) the thickness of the gap endplate edge. This was necessary since the cavity frequency changed unexpectedly from pre- to postweld, presumably because of changes in the effective dimensions due to the welding. The frequency dependence on gap width was measured to be $1.2 \pm 0.2 \mathrm{kHz} / \mathrm{mil}$.

The cavity frequencies were also tuned by inserting fixed copper slugs into the 3.87 " ports which are located $35^{\prime \prime}$ on center from the cavity shorted end (See Fig 3 ). The slugs are OFHC copper cylinders with a diameter of $3.8^{\prime \prime}$. The parts of the cylinder which protrude into the cavity have been rounded on the end and have a radius of $0.5^{\prime \prime}$. 
They are attached to water cooled Conflat ${ }^{\circledR}$ blanks and protrude nominally 1 " into the cavity. Final machining of their length sets the cavity frequency. In order to maintain a uniform temperature at high duty factor, there are 12 cooling lines each on the cavity center and outer conductors. Each set has a flow rate of $60 \mathrm{gal} / \mathrm{min}$ of $95^{\circ} \mathrm{F}$ cooling water. The outer cooling lines were Heliarc ${ }^{\circledR}$ brazed to the cavity using Sil-Fos ${ }^{\circledR}$ braze material. The lines on the inside of the ID of the center conductor were shrink-fitted with an interference of $0.040^{\prime \prime}$.

The cavity input coupler connects to a $9 \frac{3}{16}^{\prime \prime}, 50 \Omega$ transmission line through a copper and stainless steel bellows. The coupler is constructed from a conical, $12^{\prime \prime}$ diameter, 94\% alumina ceramic brazed to copper rings. The rings are welded to additional copper sections to form the coupler outer and inner conductors. The coupler $2^{\prime \prime}$ diameter inner conductor is welded to the cavity inner conductor $7.5^{\prime \prime}$ from the shorted end. A copper transition piece is welded to the cavity outer conductor and this transition is in turn welded to the outer conductor of the vacuum window assembly.

The cavity outer conductor has eight $3.87^{\prime \prime}$ diameter and four $1.65^{\prime \prime}$ diameter ports. Furnace brazed copper to stainless steel transitions are used to connect either 6 " or 2-3/4" Conflat flanges (depending on the port size) to the copper cavity body, except for one of the larger ports, which uses a copper flange for a fast tuner. Three of the larger ports located $35^{\prime \prime}$ (on center) from the cavity shorted end are used for the fixed tuning slugs which were discussed above. The four smaller ports are all located near the cavity gap end: $44^{\prime \prime}$ from the cavity short. Two of these contain the capacitively coupled field probes for measurement of cavity gap voltage. These were calibrated by simultaneously measuring the field probe power and the cavity gap voltage with an Agilent 85024A High Frequency Probe and network analyzer. A third smaller port contains the $1^{\prime \prime}$ diameter copper gap shorting slug. The slug is mounted on a linear vacuum feedthrough, which can be remotely moved into the cavity to contact the center conductor. In one of the larger ports located $3^{\prime \prime}$ from the cavity shorted end and opposite the input coupling loop, the tuner is loop-coupled to the cavity through a $2.5^{\prime \prime}$ diameter coaxial ceramic window.

At completion, each cavity weighs 4500 lbs. A photograph of the cavity is shown in Fig 4. Some additional aspects of the cavity mechanical design are discussed in 


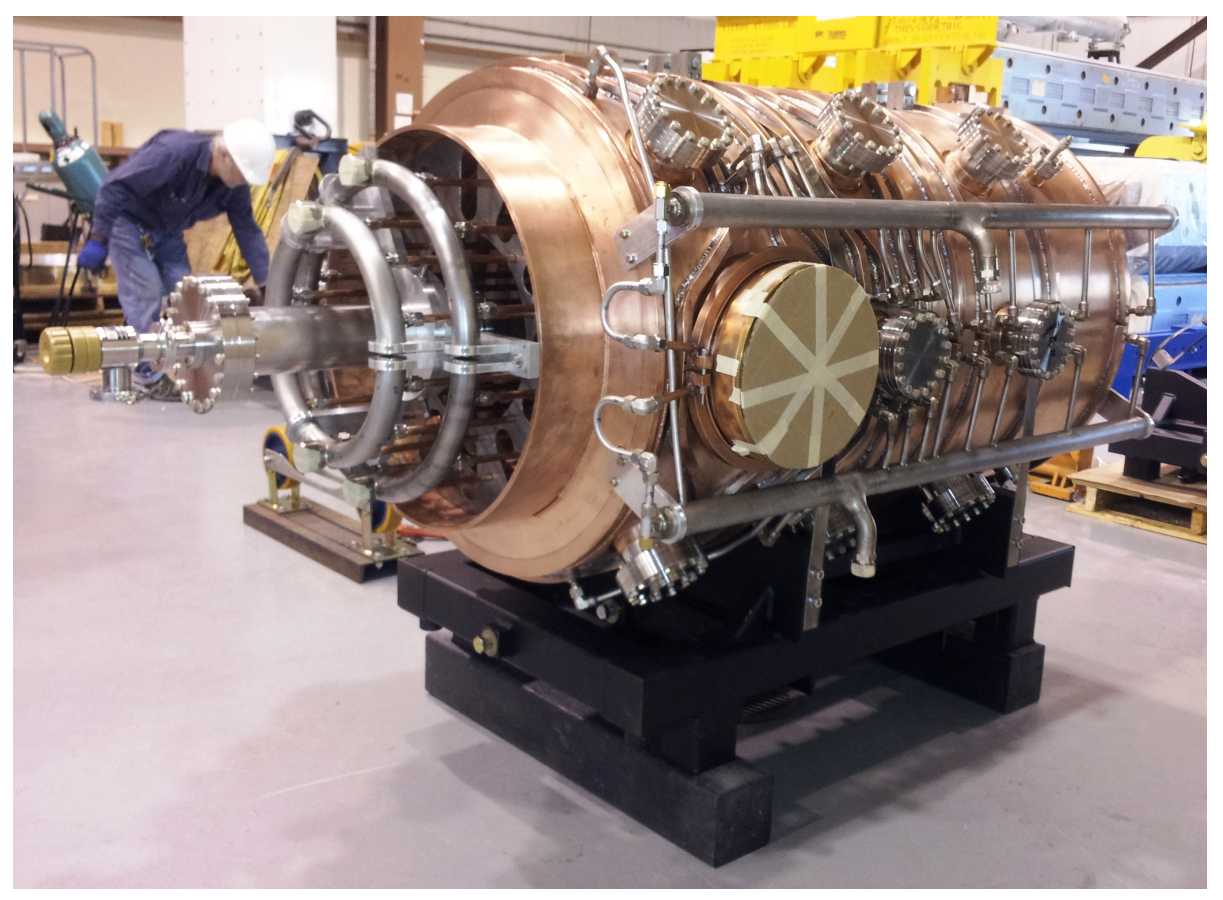

Figure 4: A photograph of one completed cavity.

[15]. Photographs of the cavity field probes, shorting slug, and input coupler window are shown in Fig 5 .

\section{Higher Order Modes}

The calculated and measured lowest frequency higher order modes of the cavity are shown in Table 2 Modes are classified as TEM ( $E$ and $B$ fields perpendicular to direction of propagation) or TE $\left(\mathrm{H}_{m n}, E\right.$ but not $B$ perpendicular to direction of propagation). The TEM modes are more of a concern because they can be more easily excited by the beam. All TM $\left(\mathrm{E}_{m n}\right)$ modes have frequencies above $2 \mathrm{GHz}$.

The frequencies of the TE modes are calculated analytically for a coaxial waveguide, assuming a line length $l$ of $50^{\prime \prime}$, and using

$$
\lambda=\frac{\lambda_{g}}{\sqrt{1+\left(\lambda_{g} / \lambda_{c}\right)^{2}}}
$$




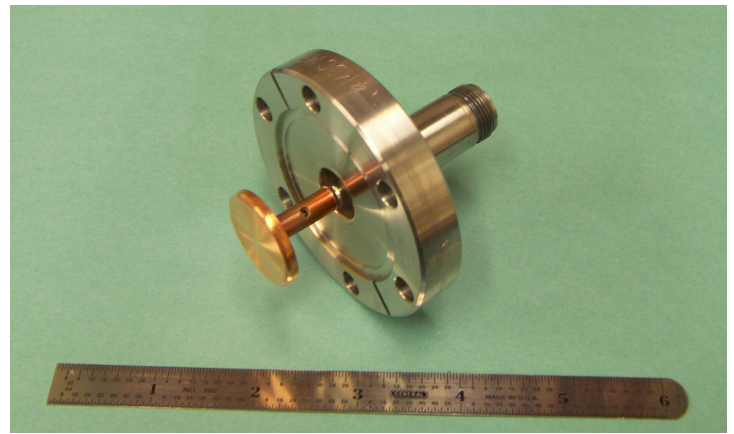

(a) Cavity field probe

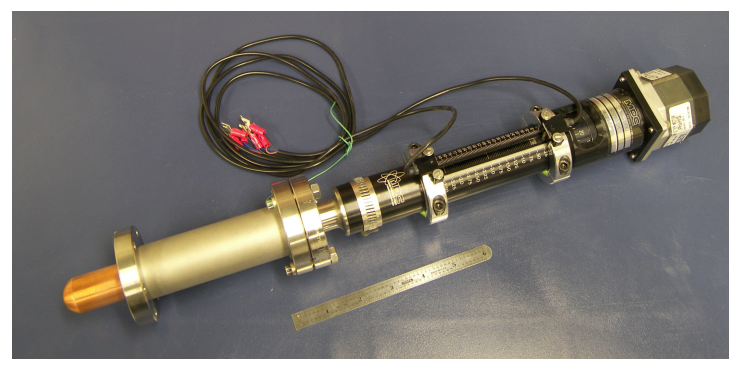

(b) Shorting slug

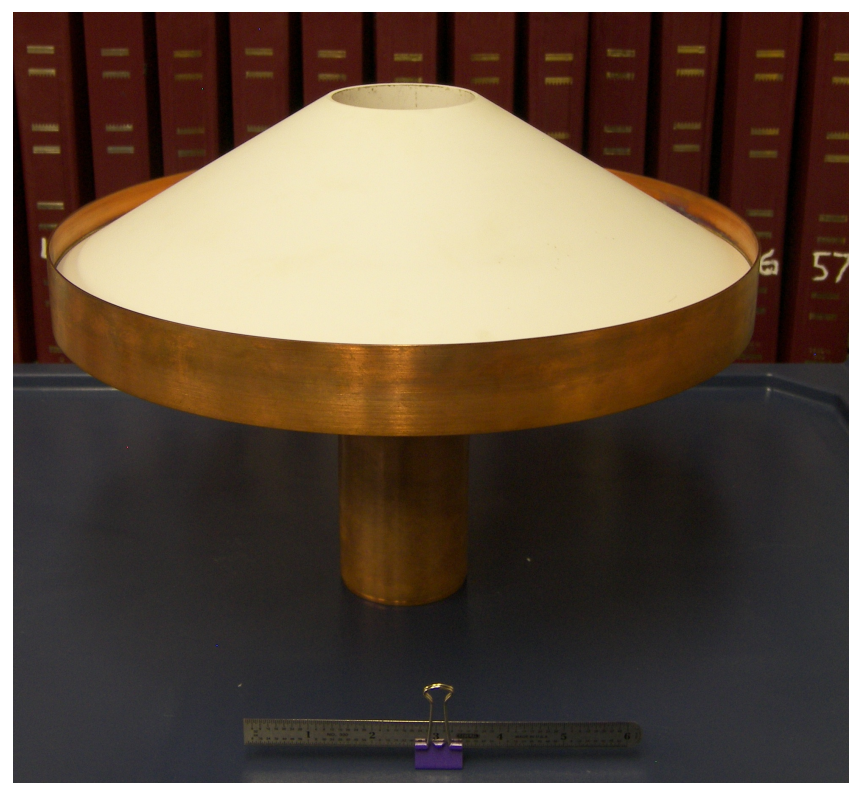

(c) Input coupler ceramic window with outer and inner conductor

Figure 5: Photographs of various cavity accessories with a 6 " ruler for scale comparison. 
where $\lambda_{g}$ and $\lambda_{c}$ are the guide and cutoff wavelengths of the coaxial line [16], and

$$
l=k \lambda_{g} / 4 \quad k=1,3,5 \ldots
$$

The TEM modes were calculated using the LANL Superfish program[17].

Modes were initially identified using a cavity model constructed from copper sheet metal. In this case, if there was an ambiguity, TEM modes could be distinguished from TE modes by using a rotatable magnetically coupled pickup loop. For TEM modes, there is no signal when the plane of the loop is perpendicular to the axis of the cavity.

The modes listed in the table were measured with a cavity in the test cave before final installation. The exact frequency of the modes will vary depending on tuner bias and the length of coaxial waveguide between the quarter wave transformer and the cavity input (i.e the setting of the variable length phase shifter - see Section 3.2). For the measurements in the cavity test cave, it was not possible to use a rotatable pickup loop to distinguish between TEM and TE modes, because the cavity was under vacuum. Instead, signals from each of the two field probes were compared. The field probes are located at $\pm 45^{\circ}$ from the input coupler, which is the main feature disturbing the azimuthal symmetry in the cavity. Thus, TEM modes will have very similar signals on each field probe, whereas many of the TE modes will not.

The 3rd harmonic TEM mode is damped by a $1 / 2^{\prime \prime}$ Heliax ${ }^{\circledR}$ quarter wave $50 \Omega$

transmission line with a capacitive pickup on the $9 \frac{3}{16}^{\prime \prime}$ transmission line (near a voltage maximum). For damping, a $5 \mathrm{~W}, 50 \Omega$ load is connected to the heliax line near the $50 \Omega$ tap point. A picture and sketch are shown in Fig.6.

\section{Tuner}

The cavity tuner makes use of perpendicularly biased garnet (a ferrimagnetic material). The DC bias field which is varied to change the effective permeability of the garnet and thus the cavity frequency, is perpendicular to the RF magnetic field. The perpendicular bias approach has been pursued with success [18][19][20][21] [22], but traditionally and most often, parallel biased ferrites such as NiZn have been used for cavity tuning. Some examples of this are in the FNAL Booster [23], Main Ring[24][25], 


\begin{tabular}{|c|c|c|c|}
\hline $\begin{array}{c}\text { Calculated } \\
\mathrm{f}(\mathrm{MHz})\end{array}$ & $\begin{array}{c}\text { Measured } \\
\mathrm{f}(\mathrm{MHz})\end{array}$ & Type & Order \\
\hline 52.809 & 52.809 & TEM & $\lambda / 4$ \\
\hline 140.36 & $\begin{array}{l}151.720 \\
154.695\end{array}$ & $\mathrm{TE}_{11}$ & $\lambda_{\mathrm{g}} / 4$ \\
\hline 154.748 & 157.780 & TEM & $3 \lambda / 4$ \\
\hline 218.081 & $\begin{array}{l}222.19 \\
226.13\end{array}$ & $\mathrm{TE}_{11}$ & $3 \lambda_{\mathrm{g}} / 4$ \\
\hline 245.228 & 247.17 & TEM & $5 \lambda / 4$ \\
\hline 261.450 & $\begin{array}{l}267.795 \\
271.630\end{array}$ & $\mathrm{TE}_{21}$ & $\lambda_{\mathrm{g}} / 4$ \\
\hline 310.186 & $\begin{array}{c}308.890 \\
312.0\end{array}$ & $\mathrm{TE}_{21}$ & $3 \lambda_{\mathrm{g}} / 4$ \\
\hline 318.485 & $\begin{array}{l}318.63 \\
323.81\end{array}$ & $\mathrm{TE}_{11}$ & $5 \lambda_{\mathrm{g}} / 4$ \\
\hline $\begin{array}{c}331.111 \\
385.8\end{array}$ & $\begin{array}{c}337.80 \\
388\end{array}$ & $\begin{array}{l}\text { TEM } \\
\mathrm{TE}_{31}\end{array}$ & $\begin{array}{l}7 \lambda / 4 \\
\lambda_{\mathrm{g}} / 4\end{array}$ \\
\hline 389.79 & $\begin{array}{c}392.44 \\
393.085\end{array}$ & $\mathrm{TE}_{21}$ & $5 \lambda_{\mathrm{g}} / 4$ \\
\hline 421 & $\begin{array}{c}428.15 \\
428.870\end{array}$ & $\mathrm{TE}_{31}$ & $3 \lambda_{\mathrm{g}} / 4$ \\
\hline 429.882 & 437.130 & TEM & $9 \lambda / 4$ \\
\hline 432.281 & 432.42 & $\mathrm{TE}_{11}$ & $7 \lambda_{\mathrm{g}} / 4$ \\
\hline 482.74 & $\begin{array}{c}470.9 \\
473.165\end{array}$ & $\mathrm{TE}_{31}$ & $5 \lambda_{\mathrm{g}} / 4$ \\
\hline 485.32 & $\begin{array}{l}475.13 \\
486.66\end{array}$ & $\mathrm{TE}_{21}$ & $7 \lambda_{\mathrm{g}} / 4$ \\
\hline 512.8 & $\begin{array}{c}516.635 \\
518.15\end{array}$ & $\mathrm{TE}_{41}$ & $\lambda_{\mathrm{g}} / 4$ \\
\hline
\end{tabular}

Table 2: Cavity Higher Order Modes, calculated and measured. For the TE modes, the two values listed in the "Measured" column correspond to two different polarizations and are different due to the cavity asymmetry. 
the CERN PS[26] and the Brookhaven AGS[27]. The saturation magnetization is substantially lower in the garnet than in the NiZn ferrite. So, operation near saturation and thus in a region of lower losses is achievable with reasonable bias field, and the nontrivial engineering problem of cooling the ferrite is eliminated. The trade-off for this is a smaller tuning range. The present cavity required a comparatively small tuning range of $10 \mathrm{kHz}$, which made the perpendicularly biased garnet a suitable choice.

The tuner is constructed from $3 \frac{1}{8}^{\prime \prime}, 50 \Omega$ copper coaxial transmission line which is magnetically coupled to the cavity through a loop. A drawing is shown in Fig. 7 . The end of the transmission line opposite the cavity is shorted and contains aluminum doped YIG (TCI Ceramics AL-400), with a saturation magnetization of 400 gauss. The effective length of the line approaches $\lambda / 2$, and the section of the line containing the garnet is immersed in a solenoidal magnetic field. By varying the solenoid bias, the garnet permeability, effective line length, and tuner resonant frequency vary. Thus the frequency of the entire coupled cavity and tuner system also varies.

\subsection{Description}

The tuner is loop coupled to the cavity through a port located $3^{\prime \prime}$ (on center) from the cavity shorted end. The coupling is through a $2.5^{\prime \prime}$ diameter coaxial ceramic window. The tuner loop area of $2.5 \mathrm{in}^{2}$ was adjusted to give a nominal coupling impedance of $50 \Omega$.

A photograph of the garnet loaded section of the tuner (the "adjustable section") is shown in Fig. 8 The garnet cylinder is $5^{\prime \prime}$ long with an outer diameter (OD) of $3^{\prime \prime}$ and an ID of $0.65^{\prime \prime}$. The diameter of the inner conductor inside of the garnet is $0.65^{\prime \prime}$, smaller than the standard inner conductor for $3 \frac{1}{8}^{\prime \prime}$ coaxial transmission line. This makes the impedance of the garnet section $50 \Omega$ when $\mu_{e}=4$. The copper inner conductor is inserted into the garnet by shrink fitting it, by cooling the center conductor in liquid nitrogen. Over the $9^{\prime \prime}$ length closest to the short, the outer conductor has been reduced from the standard $3 \frac{1^{\prime \prime}}{8}$ coax line wall thickness to $0.020^{\prime \prime}$. Both this $9^{\prime \prime}$ section and the bottom copper shorting plate have a 0.0197" wide slot machined through the copper to reduce eddy current effects. For heat removal, the outer conductor is water cooled and the adjustable section is filled with a dielectric fluid (Diala AX).The ad- 
justable section had previously been designed for use as a fast phase shifter in vector modulators operating at $325 \mathrm{MHz}$ [28].

\subsection{Solenoid}

The 112 turn (629.92 turns per meter) solenoid (with TCI Ceramics G4 ferrite flux return) was designed to work at relatively high DC current (80A), but also at high frequency ( $\sim 2 \mathrm{kHz}$ ) AC current. While the AC response is not absolutely required, the ability to tune at $2 \mathrm{kHz}$ is an advantage. Obtaining the desired DC and $\mathrm{AC}$ response necessitated the use of Litz Cable of sufficient cross section (1/0 AWG) with 270 copper strands of 24 AWG wire insulated from each other. The total strand cross section reduces the losses in the DC regime, and the insulation between strands minimizes the eddy currents in the cable for the $\mathrm{AC}$ regime. A cooling tube was wound on the outer surface of the solenoid as a bifilar coil in order to eliminate the effect of magnetic fields caused by eddy currents induced in it. A DC magnetic field simulation predicted the resulting minimum and maximum magnetic fields in the tuner garnet of $0.058 \mathrm{~T}$ and $0.081 \mathrm{~T}$, respectively, for 8000 solenoid ampere turns.

The solenoid current is supplied by a Copley Controls Model 266 amplifier which has a nominal bandwidth of $5 \mathrm{kHz}$. The tuner response is currently limited by the solenoid inductance of $1.9 \mathrm{mH}$ and the $60 \mathrm{~V}$ max/80 A max DC supply for the Copley amplifier.

\subsection{Tuner Measurements}

Tuner measurements are described in several sections. The first section describes measurements performed with the tuner detached from the cavity. These are reflection measurements of the phase shift and loss in the tuner line. This phase shift and loss directly influence the tuning range and $Q$ of the cavity-tuner system; measurements of those quantities related to the coupled system are presented in the second section. Finally, measurements of the tuner garnet effective permeability are presented. The permeability range determines the tuning range. Though the first two sets of measurements are sufficient to characterize the tuner and demonstrate its operation, it was desirable to measure this more fundamental quantity. 


\subsubsection{Tuner Phase Measurements}

The tuner alone can be most effectively characterized by the measurements shown in Fig 9, which were made with the tuner detached from the cavity. The figure shows network analyzer $S_{11}$, reflection magnitude and phase, measurements. The device under test was the adjustable section of the tuner line (see Section 6.1 and Fig. 8) with additional standard $3 \frac{1}{8}^{\prime \prime}$ coaxial line and a type $\mathrm{N}$ to $3 \frac{1}{8}{ }^{\prime \prime}$ adapter. Neglecting reflections due to discontinuities in impedance, the phase shift (from the network analyzer to the short and back) is given by:

$$
\phi_{0}+180^{\circ}+2\left(5^{\prime \prime} \frac{360^{\circ}}{\lambda_{0}} \sqrt{\mu_{\epsilon} \epsilon}\right)
$$

where $\phi_{0}$ is the phase shift in non-garnet section of the line, $\lambda_{0}$ is the free space wavelength (in inches) at the frequency of interest, and $\mu_{e}$ is the effective relative permeability. The factor of two in the third term accounts for the fact that the wave can be viewed as traveling through 5 inches of garnet, reflected at the short, and then back through five inches of garnet. Though Eq. 4 is only approximate, it makes it clear that large changes in phase correspond to large changes in $\mu_{e}$ and thus large tuning range.

The loss is the fraction of power lost in the system:

$$
\operatorname{Loss}(\mathrm{dB})=10 \log \left(P_{\text {ref }} / P_{\text {for }}\right)
$$

where $P_{\text {for }}$ is the forward power from the network analyzer and $P_{\text {ref }}$ is the power reflected back. The power lost is $P_{\text {for }}-P_{\text {ref }}$. Almost all of the power is lost in the garnet; the loss in the copper transmission line is negligible. The plot indicates that for a solenoid bias of $40 \mathrm{~A}$ and above, the losses are constant and this is a safe region of operation. Note that in the region of low losses, the phase changes less rapidly than in the high loss region (between 10 and $30 \mathrm{~A}$ ).

\subsubsection{Cavity and Tuner Measurements}

The next set of measurements quantify the operation of the cavity/tuner system as a whole. Fig. 10(a) (wide range) and Fig. 10(b) (operation range) show the frequency and the $Q$ of the cavity/tuner system as a function of solenoid bias current. The bias at 
which the largest change in frequency and a minimum in $Q$ are observed corresponds to the case where the tuner is one half wavelength long.

An additional restriction on tuner range is due to the peak voltage that can be sustained without sparking. Fig. 11 shows low level measurements of frequency and the corresponding tuner voltage. In this case, the cavity was powered with a relatively small signal. Cavity peak accelerating gap voltage and tuner peak voltage were measured simultaneously. The values were then scaled to cavity gap voltages of $100 \mathrm{kV}$ and $150 \mathrm{kV}$. Operating with a tuner voltage above the peak voltage limit for a $3 \frac{1}{8}$ " coaxial line of $13.3 \mathrm{kV}$ will cause arcing and must be avoided. Not only is arcing in the coax line undesirable, but this can also lead to high voltages near the garnet which could be destructive. Measurements indicate that in order to limit the coaxial line voltage to $13.3 \mathrm{kV}$, the minimum current levels are $32 \mathrm{~A}$ and $40 \mathrm{~A}$, for cavity peak voltages of $100 \mathrm{kV}$ and $150 \mathrm{kV}$, respectively. With no other adjustments, and taking into account the $35 \mathrm{~A}$ limit imposed due to losses in the garnet, the tuning ranges are $11.7 \mathrm{kHz}$ and $7.5 \mathrm{kHz}$ for cavity peak voltages of $100 \mathrm{kV}$ and $150 \mathrm{kV}$.

\subsubsection{Permeability Measurements}

Although the measurements in the previous sections are sufficient to characterize the tuner and show that it can perform as needed, it is desirable to measure more fundamental garnet properties. Of particular interest is $\mu_{e}$, the effective permeability, where, for the RF wave, $v=c / \sqrt{\mu_{e} \epsilon}$. This yields a better understanding of the system and also lends itself well to future cavity design using the same type of material.

To determine permeability, it was first necessary to measure the permittivity. This was done by constructing a coaxial capacitor with the garnet and measuring the capacitance using both a HP 4263A LCR meter at lower frequencies and a vector impedance meter at higher frequencies. The permittivity $\epsilon$ was measured to be 15 . Given that there is an air gap of $\approx 0.013^{\prime \prime}$ between the garnet and the outer conductor when it is placed in the tuner transmission line, the effective $\epsilon$ is 14 .

To measure $\mu_{e}$ as a function of solenoid bias, the dielectric fluid was first removed from the tuner adjustable section. The removal of the oil made interpretation of the measurements simpler by minimizing reflections at discontinuities in impedance. Mea- 
surements as described in Section 6.3.1 (Tuner Phase Measurements) were repeated. Eq. 4 is an approximation that neglects reflections at the air-garnet interface and cannot be used to accurately determine $\mu_{e}$.

Using the electronic design software by Agilent, Advanced Design System (ADS) a series a calculations (ADS simulations) were made with the model shown in Fig 12 . In the simulation, $S_{11}$ phase was calculated for 60 different values of $\mu_{e}$ in the section of coax line near the short. Then, $\mu_{e}$ vs. $S_{11}$ phase was plotted and a fit to a fourth order polynomial was performed. Simulated points and the fit are shown in Fig 13 . Then, the functional form of the fit was used to calculate the corresponding value of $\mu_{e}$ for each actual measured phase in the real data. The resulting values for $\mu_{e}$ vs. solenoid bias are shown in Fig 14 . The main advantage of this method is that the effect of reflections at the $50 \Omega$ - garnet loaded (non $50 \Omega$ ) boundary are taken into account in the phase measurement.

\subsection{Tuner Protection}

The tuner, partially due to its small size, is one of the most vulnerable parts of the cavity. Damage may be done by high power levels (heating) or overvoltage. If the solenoid bias is reduced to below $35 \mathrm{~A}$, the garnet becomes extremely lossy, so utmost care must be taken to ensure that the current remains above this level. Overvoltage is also a problem which typically causes more damage when it happens repeatedly. Thus, several interlocks are in place to shut off RF power when these conditions occur. If the current monitor on the supply indicates that the solenoid bias has dropped below $\approx 37 \mathrm{~A}$, the low level RF is tripped off. In addition, there are two "spark" detectors for the tuner. The first spark detector will trip the interlock, if, during the RF gate, the RF envelope in the tuner changes by more than $3 \mathrm{~dB} / \mu \mathrm{s}$. A second spark detector trips the interlock based on the rate of change in the phase difference between forward and reflected power (also during the RF gate), where forward and reflected power measurements are taken from a directional coupler located in the tuner $3 \frac{1}{8}{ }^{\prime \prime}$ transmission line. 


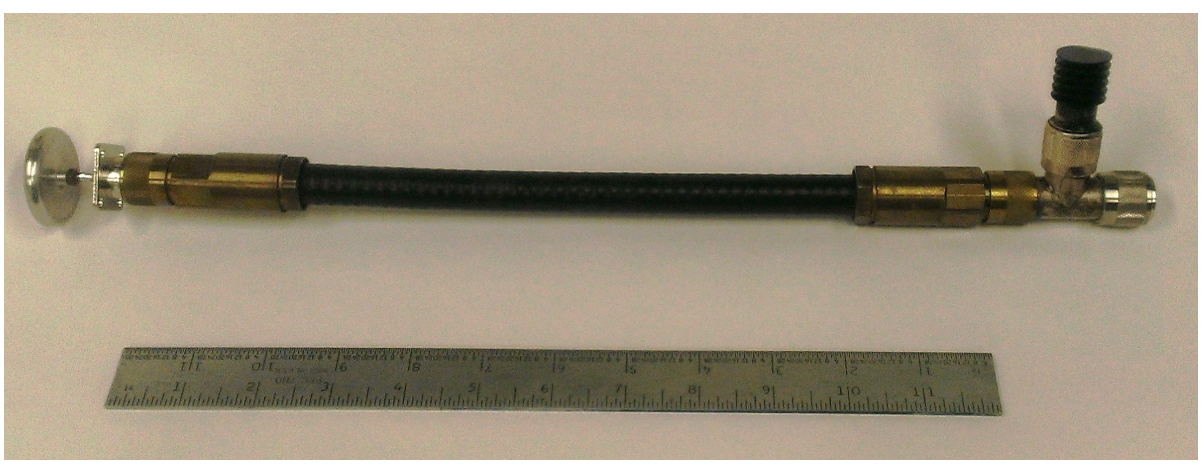

(a) Photograph of the HOM damper, removed from the $9 \frac{3}{8}{ }^{\prime \prime}$ transmission line.

9-3/8" coax

transmission line

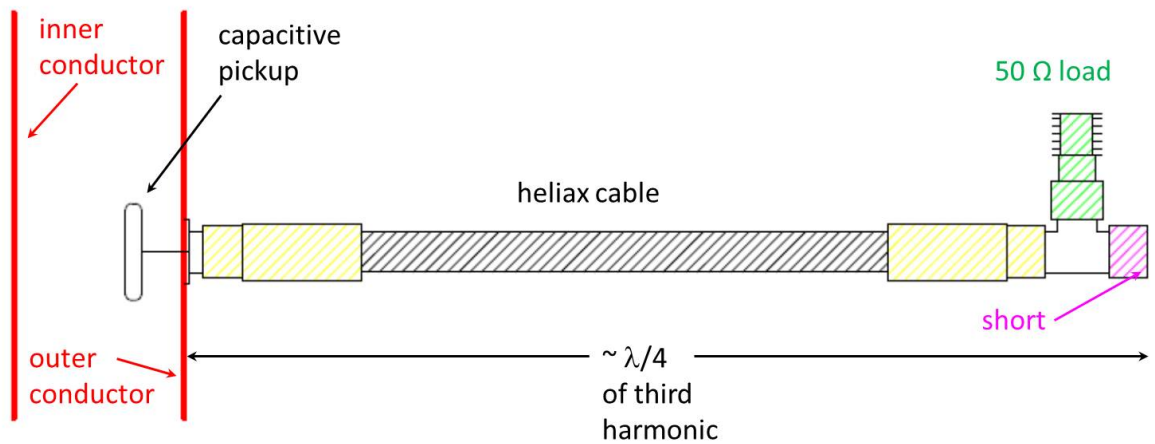

(b) Drawing of the HOM damper as mounted on the $9 \frac{3}{8} "$ transmission line.

Figure 6 


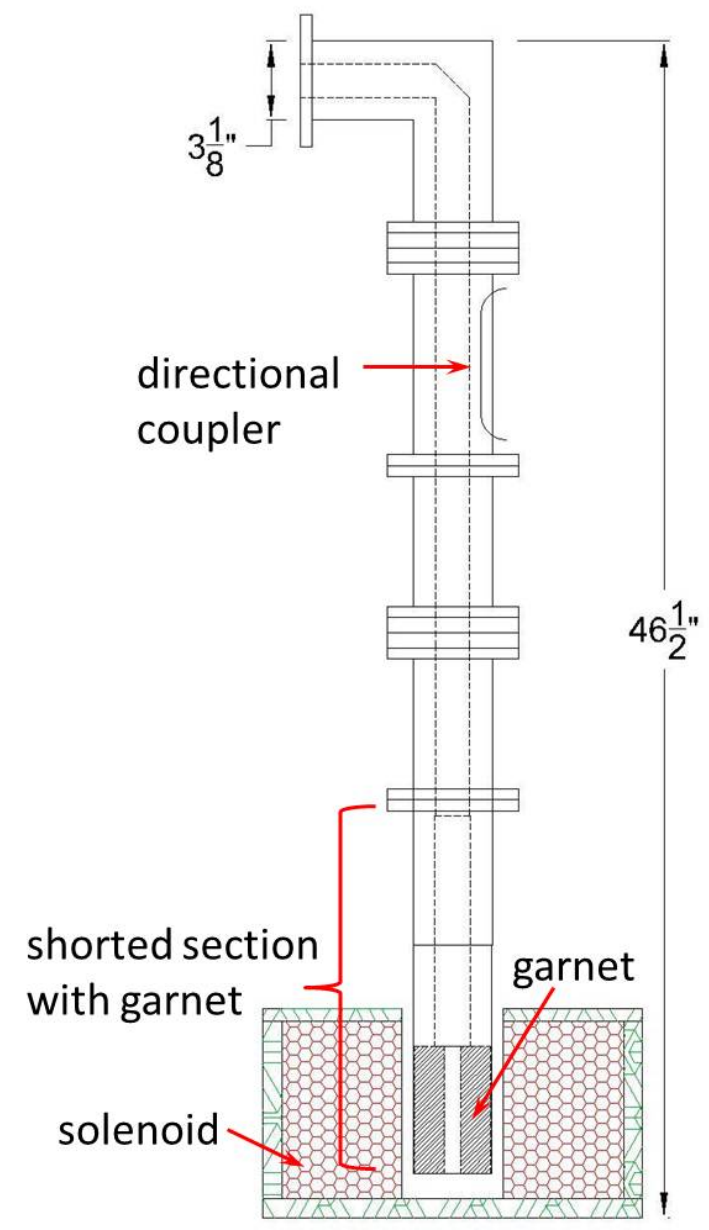

Figure 7: A drawing of the tuner. It is composed mainly of standard $3 \frac{1}{8}{ }^{\prime \prime}, 50 \Omega$ transmission line followed by the garnet section and then the short. The garnet section is immersed in a solenoidal magnetic field. 


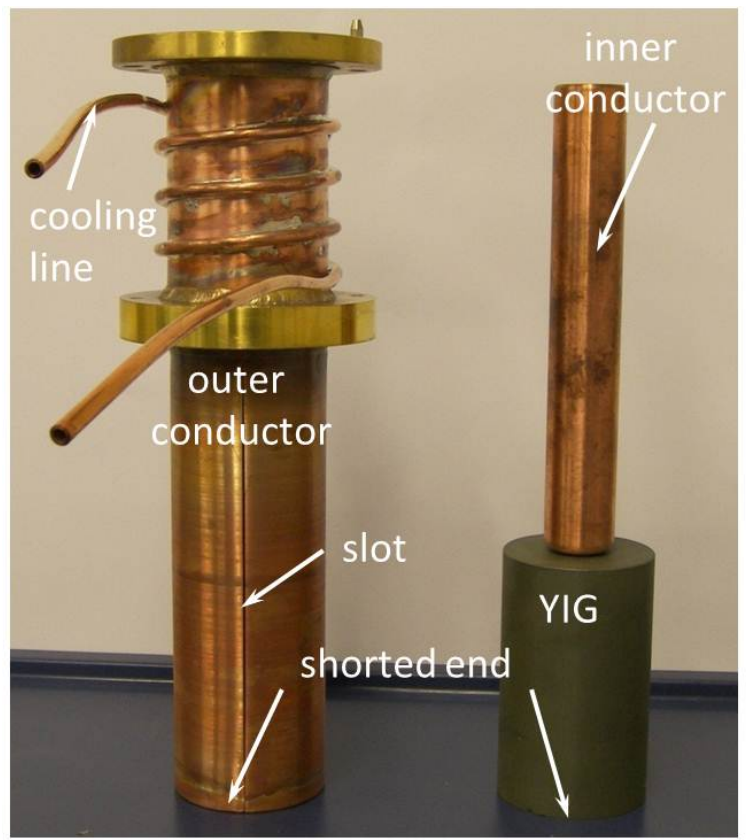

Figure 8: A photograph of a section (the "adjustable section") of the tuner. The inner conductor and garnet have been removed from the outer conductor.

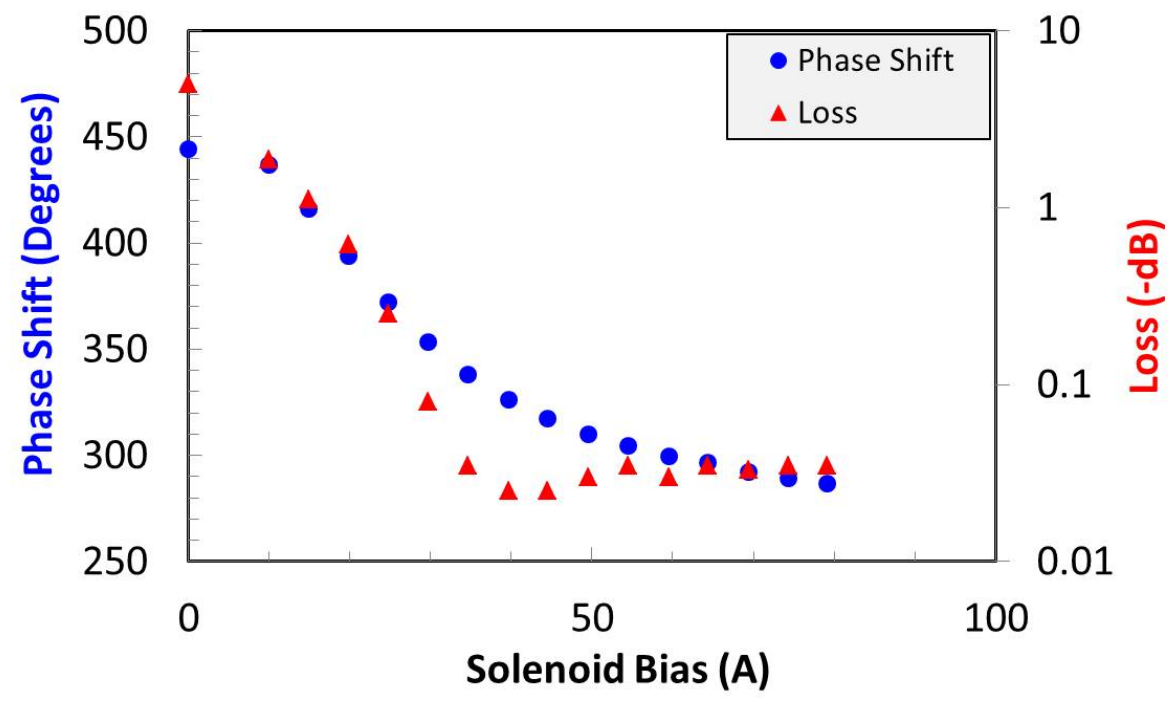

Figure 9: $S_{11}$ measurements (phase and magnitude) of a section of the tuner line. Phase shift is shown on the left axis and loss (magnitude) on the right. 


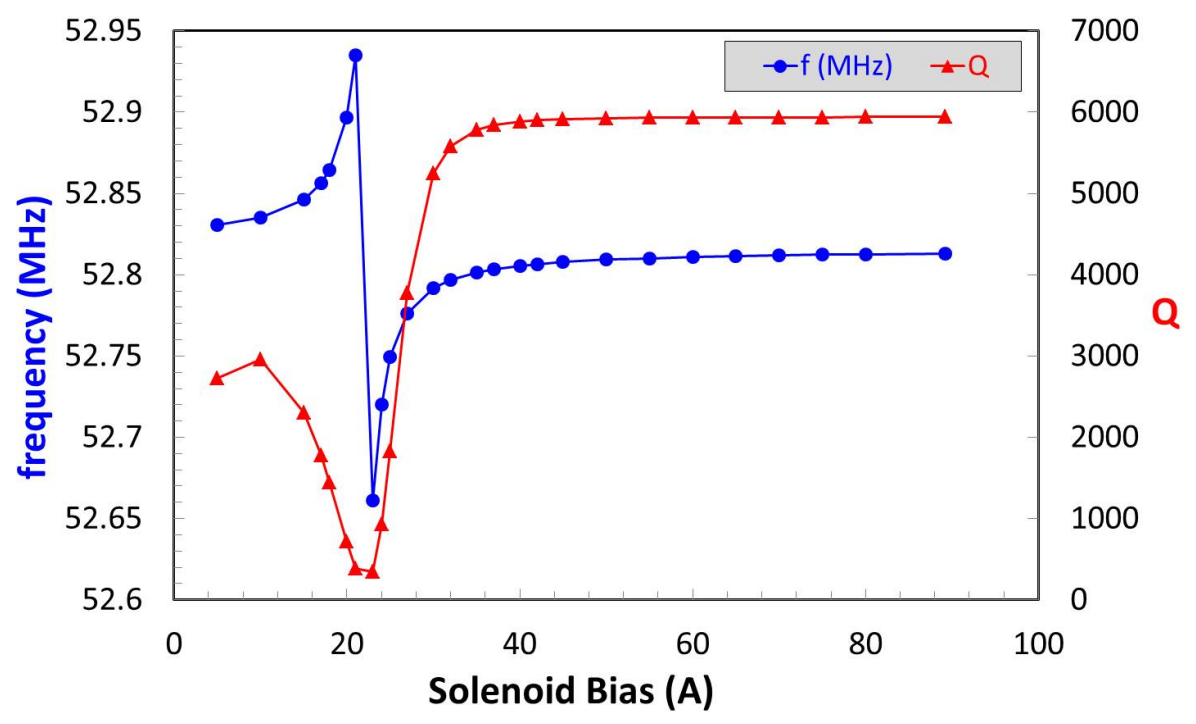

(a) Wide range plot

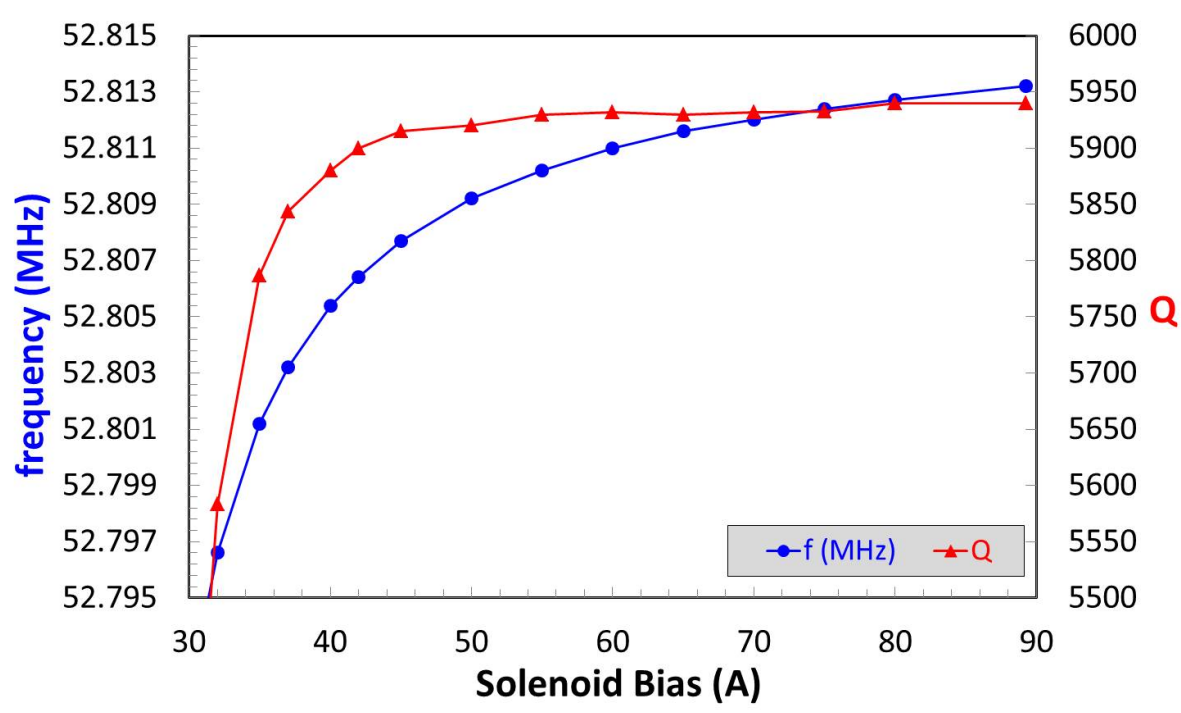

(b) Operation range plot

Figure 10: Frequency and $Q$ of the cavity-tuner system. The frequency scale is on the left axis and the $Q$ scale on the right. Two ranges in solenoid bias are shown. 


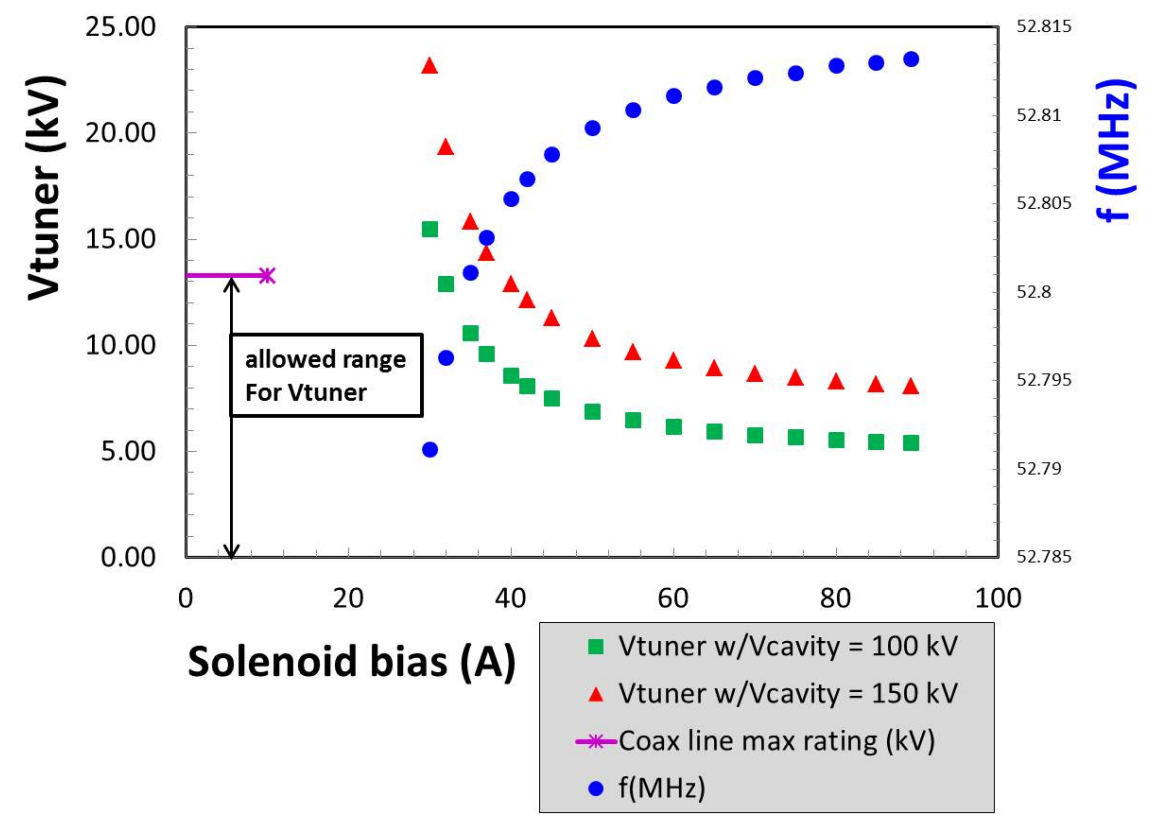

Figure 11: Tuner voltage and frequency of cavity/tuner system as a function of solenoid bias. The maximum allowed peak voltage for the tuner $3 \frac{1}{8}$ " line is also shown. 


\section{S-PARAMETERS}

S_Param

SP1

Start $=.05 \mathrm{MHz}$

Stop $=106 \mathrm{MHz}$

Step $=.001 \mathrm{MHz}$

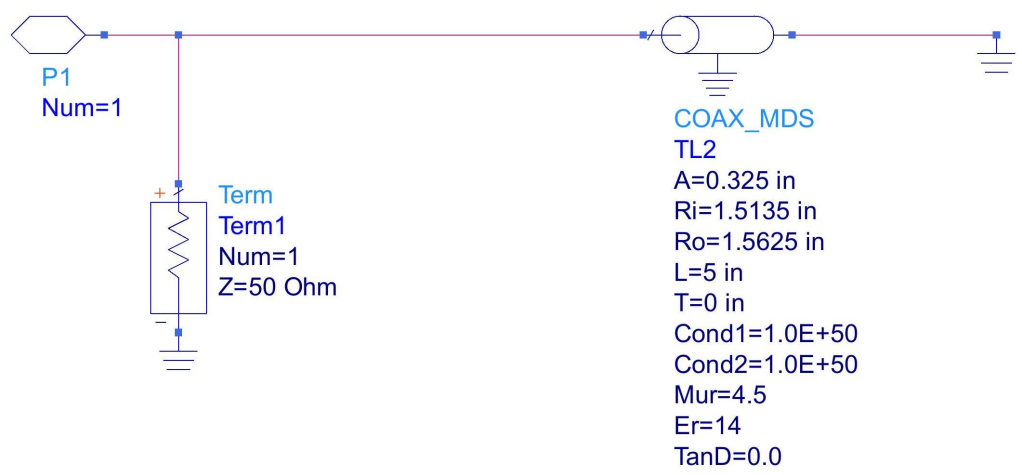

Figure 12: Agilent ADS simulation model. This calculates $S_{11}$ (with a $50 \Omega$ terminated port) into a shorted section of coaxial line with $\epsilon=14$. The permeability $\mu$ is varied. $R_{i}$ and $A$ specify the inner radius of the outer conductor and the outer radius of the inner conductor. Losses are ignored (because TanD $=0$ in this simulation) 


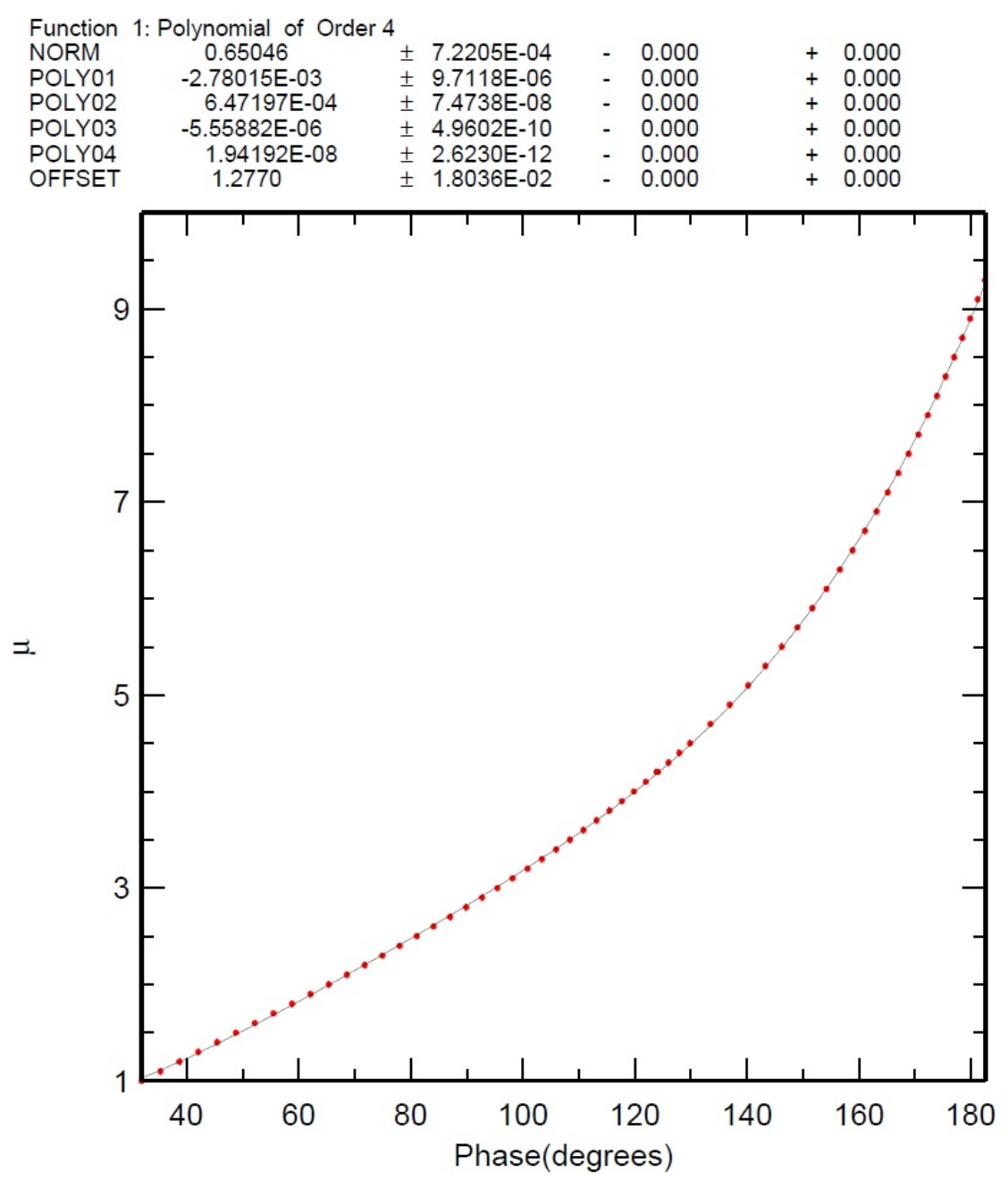

Figure 13: A 4th order polynomial fit to the results of the ADS calculation. In the simulation, $\mu$ is varied and phase is calculated. Here, the results are fit to parameterize $\mu$ as a function of phase. 


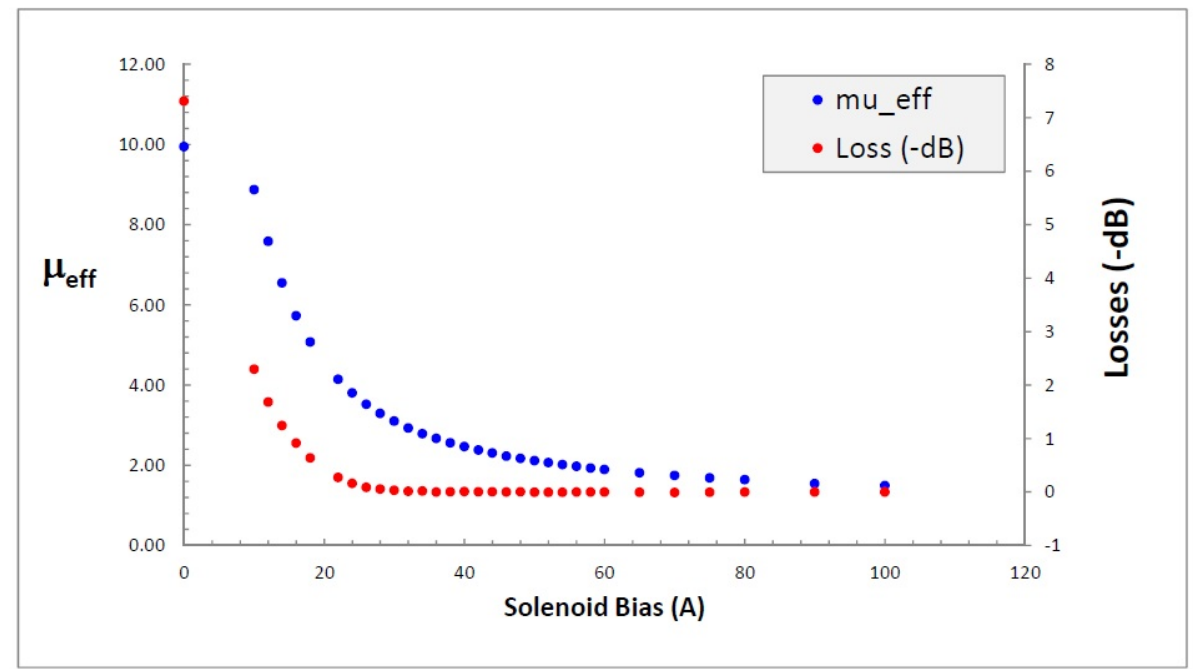

Figure 14: Effective permeability (left axis) and Losses (right axis) (Magnitude of $\mathrm{S}_{11}$ ) as a function of solenoid bias, for $f=52.809 \mathrm{MHz}$. 


\section{Testing and Operations}

\subsection{Testing}

Prior to their installation in the tunnel, two cavities were tested to full power in a cavity test cave. The cavities were first subjected to low power (up to $500 \mathrm{~W}$ ) $\mathrm{CW}$ RF for one week, to condition away multipacting between the outer and inner conductors. This was sufficient since the multipacting voltage is relatively low. The cavity was driven through the tuner port (with the tuner disconnected), which has a nominal impedance of $50 \Omega$. Multipacting is discussed in detail in [29]. The first order multipacting threshold voltage is given by

$$
V_{m p, t h}=0.039(f d)^{2}
$$

where $f$ is the frequency in $\mathrm{MHz}$ and $d$ is the spacing in inches. Multipacting was observed between the fixed tuning slugs and the center conductor $\left(d=1.5^{\prime \prime}\right)$, across the gap $\left(d=2.9^{\prime \prime}\right)$, and between the center conductor and outer conductor $\left(d=1.5^{\prime \prime}\right)$. These have calculated multipacting threshold voltages of 245,915 , and $680 \mathrm{~V}$. Note that the voltage available for multipacting $V_{m p}$ changes along the length of the cavity and is approximately $V_{\text {peak }} \sin (\pi z / 2 l)$, where $l$ is the cavity length and $z$ is the distance from the cavity shorted end. It was observed that the cavity stopped multipacting at a gap voltage of $2347 \mathrm{~V}$.

\subsection{Cavity Operation with Beam}

Two cavities have been installed in the RR. They were commissioned by first capturing beam. Later, two batches were slip stacked. On Feb 14, 2014, 12 batches of beam were successfully slip stacked, accelerated in the MI, and sent to NuMI/NOvA. Fig 15 shows this process. The Booster beam intensity is lower here than in the ultimate scenario. In that case, the Booster intensity will be $\approx 4.3 \times 10^{12}$ protons per pulse resulting in $4.9 \times 10^{13}$ protons per MI cycle to $\mathrm{NuMI} / \mathrm{NO} v \mathrm{~A}$. (This assumes $5 \%$ beam loss.) 


\subsection{Status}

A third cavity, which is nearing completion, is expected to be installed in the fall of 2014. This cavity could operate as a spare in the case where one cavity is damaged. Alternatively, it could be operated simultaneously with the other two cavities which would reduce the average power required for the cavity with the higher duty cycle. In this case, two cavities operating at half the voltage would be used for RF system A.

\section{Summary}

We have developed new RF systems and have constructed two new RF cavities for slip stacking beam in the FNAL Recycler Ring. This has made innovative reuse of RF components and of the Recycler Ring itself, which was previously used for antiproton storage and cooling. The cavities make use of several novel design features, including a perpendicularly biased garnet tuner. The system has been commissioned and has shown excellent performance in beam operations, paving the way to a record $700 \mathrm{~kW}$ on target. 


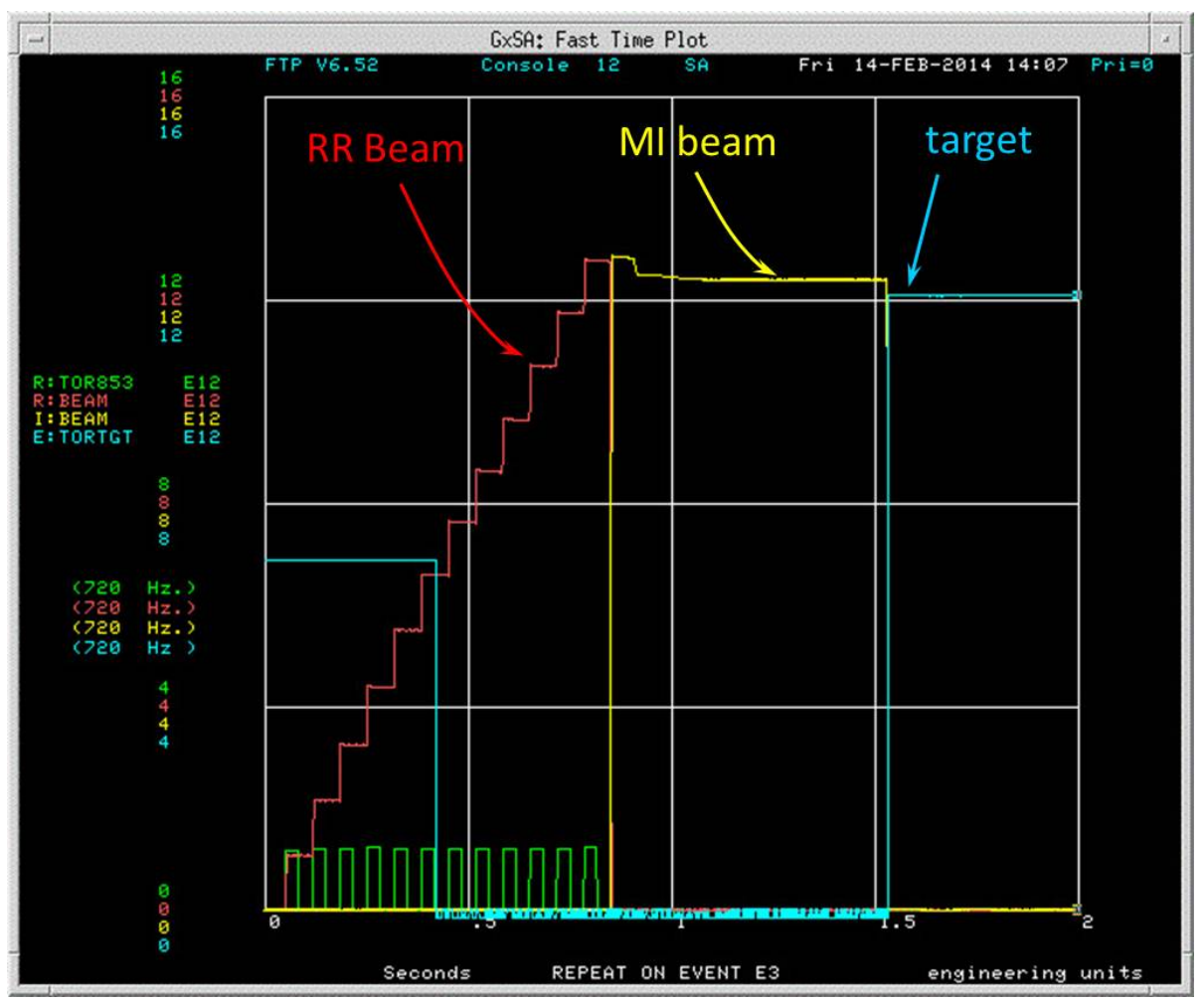

Figure 15: Slip stacking in the RR and acceleration in the MI. Beam intensity (number of protons) in the

RR, MI, and on the NuMI target are shown in red, yellow, and blue. The RR intensity is stepped as a function of time, indicating 12 separate Booster injections. The MI intensity is mostly flat, and beam remains there for the time it takes to be accelerated to $120 \mathrm{GeV}$. Finally, beam is sent to the NuMI target.

The beam is on target for $11.1 \mu \mathrm{s}$, but the target intensity as displayed above is sampled and held. 


\section{Acknowledgments}

We would like to thank everyone involved in this project. This includes, in particular, the Accelerator Physics Center for supporting our efforts, Technical Division for the design and manufacture of the solenoids for the tuners, the Accelerator Division (AD) Mechanical Support group for the cavity construction, the AD RF group for the commissioning of the high level RF systems, and the AD MI Group and Operations for the beam studies needed to achieve beam capture and slip stacking. We would also like to thank C. Y. Tan and A. Mukherjee for proofreading the manuscript.

This work is supported by Fermi Research Alliance, LLC under Contract No. DEAC02-07CH11359 with the United States Department of Energy.

[1] S. D. Holmes, V. D. Shiltsev., The legacy of the Tevatron in the area of accelerator science, Annu. Rev. Nucl. Part. Sci. 63 (2013) 435-65.

[2] S. D. Holmes, et al., Overview of the Tevatron collider complex: Goals, operations and performance, Journal of Instrumentation 6 (08).

[3] F. G. Garcia, W. Pellico, Current and planned high proton flux operations at the FNAL Booster, in: Proc. HB2012, Beijing, China, 2012, pp. 378-380.

[4] M. A. et al., Multi-physics analysis of the Fermilab Booster RF cavity, in: Proc. IPAC 2012, New Orleans, USA, 2012, pp. 3347-3349.

[5] C. Tan, et al., The $750 \mathrm{keV}$ RFQ injector upgrade, Tech. Rep. Beams-doc-3646v14, Fermilab (2013).

URL http://beamdocs.fnal.gov/AD-public/DocDB/ShowDocument? docid $=3646$

[6] R. P. and NOvA Collaboration, Status of the NOvA experiment, Journal of Physics: Conference Series 120 (5).

[7] D. Michael., The MINOs experiment, Progress in Particle and Nuclear Physics 48 (1) (2002) 99-109. 
[8] D. Ayres, et al., The NOvA technical design report, Chapter 8, Tech. Rep. FERMILAB-DESIGN-2007-01, Fermilab (2007).

[9] D. Boussard, "Beam Loading", in Handbook of Accelerator Physics and Engineering, World Scientific, Singapore, 2002, p. 110.

[10] K. Seiya, et al., Progress in multi-batch slip stacking in the Fermilab Main Injector and future plans, in: Proc. PAC 2009, 2009, pp. 1424-1426.

[11] D. Boussard, Y. Mizumachi, Production of beams with high line-density by azimuthal combination of bunches in a synchrotron, IEEE Trans. Nucl. Sci. NS26 (1979) 3623.

[12] P. Wilson, J. Griffin., High energy electron linacs; application to storage ring RF systems and linear colliders (appendix), in: AIP Conf. Proc., Vol. 87, 1982, p. 450 .

[13] H. Elnaiem, Prototype NOvA RF cavity for the Fermilab Recycler Ring (2009). URL https://indico.fnal.gov/getFile.py/access?contribId= 10\&res Id=1\&materialId=paper\&conf $I d=2665$

[14] J. Reid, H. Miller, A $200 \mathrm{kw}$ power amplifier and solid state driver for the Fermilab Main Injector, in: Proc PAC 1995, 1996, pp. 1544-46.

[15] C. Ader, D. Wildman, Design methodology and considerations for $\mathrm{NO} v \mathrm{~A} 53 \mathrm{MHz}$ RF cavities, in: Proc. IPAC 2010, 2010, pp. 4158-60.

[16] N. Marcuvitz., Waveguide handbook, The Institute of Engineering and Technology, London, 2009, pp. 72-80.

[17] L. A. N. Lab, Superfish, http://laacg.lanl.gov/laacg/services/ download_sf.phtml.

[18] S. M. Hanna, et al., YIG tuners for RF cavities, IEEE Transactions on Magnetics 28 (5) (1992) 3210-3212. 
[19] S. M. Hanna., Applications of ferrites and ferromagnets in tuning RF cavities for accelerators, Vol. 75, 1994, pp. 5625-5627.

[20] R. L. Porier., Perpendicular biased ferrite tuned cavities, in: Proc PAC 1993, 1993, pp. 753-757.

[21] L. Earley, et al., A high-Q ferrite-tuned cavity, IEEE Trans. Nucl. Sci. NS-30 (4).

[22] R. M. Hutcheon, A perpendicularly-biased ferrite tuner for the $52 \mathrm{MHz}$ Petra II cavities, in: Washington 1987, Accelerator engineering and technology, Vol. 3, 1987, pp. 1543-45.

[23] E. L. Hubbard, Booster synchrotron, Tech. Rep. FERMILAB-TM-0405, Fermilab (1973).

[24] J. E. Griffin, Q. A. Kerns, NAL main ring cavity test results, in: Proc. 4th IEEE Particle Accelerator Conference, 1971, pp. 241-243.

[25] Q. A. Kerns, B. R. Sandberg, The RF ferrite-testing program at NAL, in: Proc. 4th IEEE Particle Accelerator Conference, 1971, pp. 244-245.

[26] R. Regenstreif, The CERN proton synchrotron, Tech. Rep. CERN 59-29, CERN (1959).

[27] G. Rakowsky., RF accelerating cavities for the AGS conversion, IEEE Trans. Nucl. Sci NS-14 (3) (1967) 315-319.

[28] R. Madrak, D. Wildman, High power $325 \mathrm{MHz}$ vector modulators for the Fermilab High Intensity Neutrino Source (HINS), in: Proc. LINAC 2008, 2008, pp. 996-998.

[29] B. H. Smith, Radiofrequency system of the Berkeley 88-inch Cyclotron, Nuclear Instrum. Methods 18,19 (1962) 184-193. 